Conquering the complexity of p53

\author{
Laura D Attardi \& Ronald A DePinho
}

New evidence from a Trp53 'knock-in' mouse model suggests that p53-dependent cell cycle checkpoint control accompanied by maintenance of genome stability is important for keeping tumor growth in check.

Malignant transformation represents the phenotypic end-point of multiple genetic aberrations acting in concert to endow cancer cells with a large assortment of biological capabilities $^{1}$. p53 is a sequence-specific transcription factor that stands at the nexus of sensing and integrating diverse growth and survival signals and converting this information into highly coordinated gene expression patterns required to maintain cellular homeostasis and tumor-free survival of the organism $^{2}$. Much research effort is devoted to dissecting the relative in vivo roles of the downstream signaling functions of p53 by either inactivating specific signaling surrogates (i.e., particular p53 target genes) or altering p53 itself such that it retains only a subset of its specific activities. On page 63 of this issue, Lozano and colleagues use the latter approach to show the importance of p53 cellcycle regulation in tumor suppression in vivo.

Liu et al. engineered a Trp53 knock-in mutation in the mouse germ line $(515 \mathrm{G} \rightarrow \mathrm{C}$, resulting in the amino acid substitution $\mathrm{R} 172 \mathrm{P})$, analogous to a human tumor-derived mutant shown previously to be incompetent in activating apoptosis yet capable of inducing arrest in the G1 phase of the cell cycle ${ }^{3}$. The probable basis for this selective activity is that the mutant protein retains the capacity to transactivate the gene $C d k n 1 a$, encoding the

Laura D. Attardi is in the Departments of Radiation Oncology and Genetics, Stanford University School of Medicine, Stanford, California 94305, USA. Ronald A. DePinho is in the Department of Medical Oncology, DanaFarber Cancer Institute and the Departments of Medicine and Genetics, Harvard Medical

School, Boston, Massachusetts 02115, USA. e-mail: attardi@stanford.eduor ron_depinho@dfci.harvard.edu. p21 cyclin-dependent kinase inhibitor, but not apoptotic target genes, such as $B a x^{4}$ (Fig. 1). Correspondingly, cells derived from $\operatorname{Trp53} 3^{515 \mathrm{C} / 515 \mathrm{C}}$ mice were partially competent for cell cycle arrest but completely defective in apoptosis in response to DNA damage. With one primary function of p53 compromised, Liu et al. could now assess the requirement and relative contribution of the apoptotic function of $\mathrm{p} 53$ to tumor suppression in vivo.

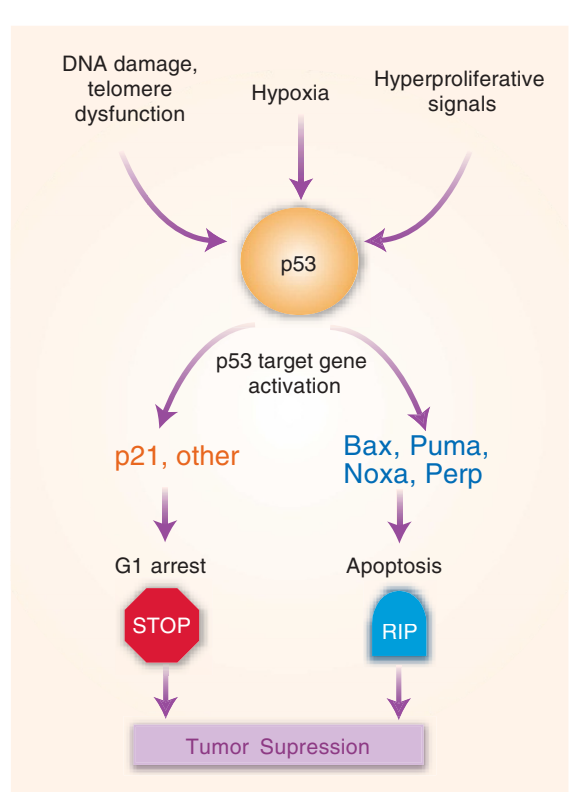

Figure 1 A number of cellular stresses, including DNA damage, hypoxia and hyperproliferative signals, activate p53 to stimulate target gene expression. p53 induces genes encoding p21 and some other proteins to implement a G1 arrest response and genes encoding Bax, Puma, Noxa and Perp to activate the apoptotic pathway. The particular downstream pathway activated by p53 is influenced by cellular context, and both pathways contribute to tumor suppression.
In marked contrast to the early-onset thymic lymphoma phenotype of mice completely deficient in p53 (ref. 5), tumorigenesis was substantially delayed in $\operatorname{Tr} p 53^{515 \mathrm{C} / 515 \mathrm{C}}$ mice, with thymic lymphoma development strongly inhibited. Moreover, the lymphomas that eventually emerged in Trp53515C/515C mice had notably benign diploid cytogenetic profiles, in contrast to the aneuploidy present in tumors derived from $\operatorname{Tr} p 53^{--}$mice. These genetic data support the view that the cell cycle checkpoint function of p53 and maintenance of genomic stability have prominent roles in the suppression of thymic lymphoma development in vivo.

\section{Damned dogma!}

The prevailing view in the cancer field is that activation of apoptosis is the dominant mode of tumor suppression by $\mathrm{p} 53$. This idea results from several different lines of evidence. First, original reports of mice deficient in p21 indicated that these mice had a compromised G1 arrest response but did not reproduce the cancer-prone phenotype of p53-null mice ${ }^{6-8}$. Second, ectopic cell cycle entry caused by inactivation of retinoblastoma $(\mathrm{Rb})$ function in the mouse by mutation ${ }^{9}$ or viral oncoprotein sequestration ${ }^{10}$ engenders a p53-dependent apoptotic response. In an extension of this work to a model of epithelial carcinogenesis, viral oncoprotein inactivation of $\mathrm{Rb}$ family function provokes a neoplastic phenotype typified by high mitotic index and accompanying apoptosis that, on a p53-null background, yields a rapidly progressive, apoptosis-resistant tumor phenotype. Moreover, by using mice lacking the proapoptotic protein Bax, this model provided compelling genetic evidence that apoptosis contributes substantially to tumor suppression ${ }^{11}$. Third, use of a 
mouse model of Myc-induced B-cell lymphomagenesis showed that kinetics of Mycinduced lymphoma in mice defective in specific components of the apoptotic pathway, such as caspase- 9 , are indistinguishable from those observed in the absence of p53 (ref. 12). The conclusions drawn from these studies have been bolstered by the existence of human tumor-derived p53 mutants compromised in apoptosis but not arrest function-arguing for the crucial importance of inactivating apoptotic functions during the course of human tumorigenesis.

Both cell cycle regulation and the induction of apoptosis are fundamental to the action of p53 in the mouse. Which activity is more relevant is probably context-dependent, with cell cycle regulation seeming to be key in thymocytes and apoptosis prevailing in other cell types, such as B cells. One prediction from Liu et al. is that mice deficient in p53 apoptotic target genes would not be prone to thymic lymphoma. The concept that apoptotic deficiency is not sufficient for thymic lymphomagenesis represents a change from previous thinking; because thymocytes undergo a clear p53-dependent apoptotic response on DNA damage ${ }^{13}$, there is a longstanding belief that the predisposition of $\mathrm{p} 53$ deficient mice to thymic lymphoma results from defects in this apoptotic program.

These studies also raise the question of why $C d k n 1 a^{-/-}$mice, which have a compromised G1 arrest response, are not prone to thymic lymphoma. This condition may relate to the fact that p21-deficient cells are only partially defective in the G1 arrest checkpoint, and that the activity of another, unknown p53-regulated cell cycle arrest target gene can provide sufficient cell cycle regulation to prevent the malignant transformation of thymocytes. Another, not mutually exclusive, explanation for limited tumorigenesis in $C d k n 1 a^{-/-}$mice is developmental or somatic compensation by functionally related cyclindependent kinase inhibitors, similar to what has been described recently for the Rb family ${ }^{14}$.

\section{Vive la différence}

Among the remaining issues is the question of what triggers p53 activation to prevent thymic lymphoma development. p53 is activated by any of a number of stresses, including DNA damage, hyperproliferative signals and hypoxia. The signals that trigger p53 activation and, by extension, the downstream pathway activated by p53 may be fundamentally different in mice and humans. For example, unlike mouse cells, which have very long telomeres, human cells have relatively short telomeres and therefore suffer severe consequences on telomere attrition. Telomere-based crisis, in which critically short telomeres result in breakage-fusionbridge cycles, is accompanied by p53 activation and a prominent apoptotic response in many human cell types ${ }^{15}$. In certain human cell types, there may be a strong selection pressure specifically against the apoptotic function of $\mathrm{p} 53$, which could explain how human tumor mutants in which the apoptotic function has been inactivated might have arisen. A prediction of this idea would be that the $\operatorname{Trp} 53^{515 C}$ allele in the context of telomere-based crisis would render mice very cancer-prone in certain compartments, a hypothesis that is now testable through the use of telomerase-deficient $\operatorname{Tr} p 53^{515 \mathrm{C} / 515 \mathrm{C}}$ compound mutant mice.

p53 itself is a difficult target for cancer therapy given its active role in promoting aging when inappropriately activated. Consequently, depending on the tumor type, it may be most productive to selectively target only a specific aspect of p53 function to mollify accelerated aging in normal tissues, particularly in the setting of conventional therapy. Defining the specific signaling surrogates involved in tumor maintenance is a good first step toward designing well-tolerated and effective therapies for the many cancers related to p53 mutations.

1. Hanahan, D. \& Weinberg, R.A. Cell 100, 57-70 (2000).

2. Vousden, K.H. \& Lu, X. Nat. Rev. Cancer 2, 594-604 (2002).

3. Liu, G. et al. Nat. Genet. 36, 63-68 (2004).

4. Ludwig, R.L., Bates, S. \& Vousden, K.H. Mol. Cell. Biol. 16, 4952-4960 (1996).

5. Attardi, L.D. \& Jacks, T. Cell. Mol. Life Sci. 55, 48-63 (1999).

6. Brugarolas, J. et al. Nature 377, 552-557 (1995).

7. Deng, C., Zhang, P., Harper, J.W., Elledge, S.J. \& Leder, P. Cel/ 82, 675-684 (1995).

8. Martin-Caballero, J., Flores, J.M., Garcia-Palencia, P. \& Serrano, M. Cancer Res. 61, 6234-6238 (2001).

9. Morgenbesser, S.D., Williams, B.O., Jacks, T. \& DePinho, R.A. Nature 371, 72-74 (1994).

10. Symonds, H. et al. Cell 78, 703-711 (1994).

11. Yin, C., Knudson, C.M., Korsmeyer, S.J. \& Van Dyke, T. Nature 385, 637-640 (1997).

12. Schmitt, C.A. et al. Cancer Cell 1, 289-298 (2002).

13. Lowe, S.W., Schmitt, E.M., Smith, S.W., Osborne, B.A. \& Jacks, T. Nature 362, 847-849 (1993).

14. Sage, J., Miller, A.L., Perez-Mancera, P.A., Wysocki, J.M. \& Jacks, T. Nature 424, 223-228 (2003).

15. Chin, L. et al. Cel/ 97, 527-538 (1999).

\section{White meat or dark?}

\section{Ava E Brent \& Clifford J Tabin}

\section{In zebrafish, slow twitch muscle is specified from a somitic muscle precursor pool by local Hedgehog (Hh) signals. A new study identifies the transcription factor Blimp-1 as a key downstream mediator in this process.}

\begin{abstract}
"White meat or dark?" is a familiar question asked around holiday tables. But what exactly makes meat white or dark? Dark meat, composed primarily of slow twitch muscle fibers, is specialized for extended exertions (standing, walking, slow swimming) and gets the

Ava E. Brent and Clifford J. Tabin are in the Department of Genetics, Harvard Medical School, 77 Avenue Louis Pasteur, Boston, Massachusetts 02115, USA.

e-mail: tabin@genetics.med.harvard.edu
\end{abstract}

consistent energy it needs from its high myoglobin content. By contrast, fast muscle, the main component of white meat, fuels quick bursts (sprinting, short flights) and contains less myoglobin.

Because chickens and turkeys stand or roam about and rarely fly, their leg meat is dark while their breast and wing meat is white. Conversely, wild birds, such as ducks, which frequently fly, have dark breast and wing meat. As your holiday guests feast on their turkey, this may be enough information for them. But for authors Philip Ingham, Sudipto Roy and colleagues, the question of white meat or dark led to a fascinating investigation into how slow and fast muscle is established during zebrafish embryogenesis and to the identification of a genetic switch responsible for determining muscle fiber fate ${ }^{1}$.

\section{Instructive cues}

In the vertebrate embryo, skeletal muscle arises from somites, segmented blocks of mesoderm lying on either side of the notochord and 